compare these rates to those observed in pregnant women outside of jail; and to verify the maternal factors associated with syphilis infection during pregnancy in free and incarcerated women.

Methods We used data from two nationwide studies conducted during the period 2011-2014. The "Birth in Brazil" study included 23894 free women cared for in 266 hospitals. The "Maternal and Infant Health in Prisons" study included 495 incarcerated pregnant women or mothers living with their children, according to a census conducted in 33 female prisons. The same case definitions and data collection methods were used in both studies. We used the Chi square test to compare the characteristics of incarcerated and free women with a significance of 0.05 .

Results For incarcerated women, the estimated prevalence of syphilis during pregnancy was $8.7 \%$ (IC 95\% 5.7-13.1) and for HIV infection 3.3\% (IC 95\% 1.7-6.6), rates almost 7 times greater than that found in free women. The estimated MTCT of syphilis was $66.7 \%$ (IC 95\% 44.7-83.2) and the incidence of congenital syphilis at birth was 58.1 per 1000 living newborns (IC 95\% 40.4-82.8), rates two and 12.6 times higher than in free women, respectively. Incarcerated women showed greater social vulnerability and worse results in all the evaluated antenatal indicators. Syphilis infection was associated with social vulnerability in free women, but not in incarcerated women.

Conclusion Incarcerated women had a higher prevalence of syphilis and HIV infection during pregnancy, lower quality of antenatal care and higher levels of social vulnerability. Health initiatives in the prison system are necessary to reduce healthcare inequalities and should include adequate antenatal and birth care with opportune diagnosis and treatment of infected pregnant women as recommended in national and international guidelines.

\section{P3.196 SEXUAL NETWORK DRIVERS OF HIV AND HERPES SIMPLEX VIRUS TYPE 2 (HSV-2) TRANSMISSION: A COMPARATIVE MATHEMATICAL MODELLING ANALYSIS}

${ }^{1}$ Ryosuke Omori, ${ }^{2}$ Laith J Abu-Raddad. 'Hokkaido University, Sapporo - Japan; ${ }^{2}$ Weill Cornell Medicine, Qatar, Cornell University, Doha, Qatar

10.1136/sextrans-2017-053264.431

Introduction HIV and herpes simplex virus type 2 (HSV-2) infections are sexually transmitted and propagate in sexual networks. Our study's objectives were to quantify effects of key network statistics on infection transmission, and extent to which HSV-2 prevalence is predictive of HIV prevalence.

Methods An individual-based Monte Carlo simulation model was constructed to describe sex partnering and infections transmission. The model was parametrized with current and representative natural history, transmission, and sexual behaviour data. Correlations were assessed on model outcomes and multiple linear regressions were conducted to estimate adjusted associations and effect sizes.

Results HIV prevalence was most often lower than a third of HSV-2 prevalence. HIV and HSV- 2 prevalences were associated with a Spearman's rank correlation coefficient of 0.64 (95\% CI: 0.58-0.69). Collinearities among network statistics were detected, most notably between concurrency versus mean and variance of number of partners. Controlling for confounding, unmarried mean number of partners (or alternatively concurrency) were the strongest predictors of HIV prevalence. Meanwhile, unmarried and married mean and variance of number of partners (or alternatively concurrency), and clustering coefficient were the strongest predictors of HSV-2 prevalence. HSV-2 prevalence was a strong predictor of HIV prevalence by proxying effects of network statistics.

Conclusion Network statistics drive similar and differential effects on HIV and HSV-2 transmission. HIV prevalence reflected primarily mean and variance of number of partners, but HSV-2 prevalence was affected by a range of network statistics. HSV-2 prevalence can be used to predict a population's HIV epidemic potential, thereby informing HIV prevention interventions.

\section{P3.197 QUALITY OF LIFE OF PATIENTS LIVING WITH HUMAN IMMUNODEFICIENCY VIRUS INFECTION: EVIDENCE FROM SOUTH INDIA}

Sabin Siddique. Yenepoya University, Mangalore, India

\subsection{6/sextrans-2017-053264.432}

Introduction With anti-retroviral therapy (ART) for human immunodeficiency virus infection (HIV) coming into picture, quality of life (QOL) has gained importance. Knowledge on the factors affecting QOL would be helpful in making important policy decisions and health care interventions. The aim of this study is to assess the quality of life of people living with HIV (PLWH) and to identify the factors influencing their QOL.

Methods The study was done among 100 PLWH attending a tertiary care hospital, and three Non-Governmental Organisations at Calicut, Kerala, India, from June 2011 to May 2014. QOL was assessed using HIV specific World Health Organisation Quality Of Life scale (WHOQOL-HIV) - BREF questionnaire which has six domains (physical, psychological, level of independence, social relationships, environment and spirituality/religiousness/personal belief). Social support and stigma were measured using "Multidimensional Scale of Perceived Social Support" and "HIV Stigma Scale," respectively, using Likert Scale. Factors influencing QOL were identified using backward stepwise multiple linear regression with the six domain scores as the dependent variables.

Results Male: Female ratio was $1: 1 \%$ and $58 \%$ were in early stage of the disease (stage I/II). Psychological and SRPB (Spirituality Religiousness and Personal Beliefs) domains were the most affected domains. All the regression models were statistically significant $(p<0.05)$. The determination coefficient was highest for the social relationship domain (57\%) followed by the psychological domain (51\%). Disease stage and perceived social support significantly influenced all the domains of WHOQOL. Younger age, female gender, rural background, shorter duration of HIV, non-intake of ART and greater HIV related stigma were the high risk factors of poor QOL.

Conclusion Interventions such as ART, family, vocational and peer counselling would address these modifiable factors influencing QOL, thereby improving the QOL of PLWH. 